\title{
Síndrome de Münchhausen
}

\author{
Münchhausen syndrome
}

\author{
Soraya Aparicio-Turbay, Camilo Alvarado-Castro, \\ Efraín Noguera-Alfonso • Bogotá D.C. Colombia.
}

\section{Resumen}

Introducción: síndrome de Münchhausen es un subtipo de trastorno facticio grave y crónico, que se caracteriza por la producción de mentiras patológicas ("pseudología fantástica") y una conducta errante, de médico en médico, fingiendo una enfermedad física.

Objetivo: presentar el caso de un paciente con hipoglicemia refractaria secundaria al uso de insulina exógena en el contexto de un síndrome de Münchhausen.

Discusión: el paciente presentó una sumisión inusual a las hospitalizaciones y a las pruebas diagnósticas, las cuales confirmaron la administración exógena de insulina para desencadenar los síntomas. Los rasgos paranoides y la mitomanía observada se asocian a un estado larvado, crónico y delirante, propio de síndrome de Münchhausen.

Conclusión: el síndrome de Münchhausen es una condición psiquiátrica difícil de diagnosticar y que constituye un importante reto para el clínico, es poco reconocido, afecta la relación médicopaciente y presenta un alto costo para el sistema de salud debido al uso de innecesaria de baterías diagnósticas y procedimientos terapéuticos. (Acta Med Colomb 2019; 44:43-46).

Palabras clave: síndrome de Münchhausen, trastorno facticio, hipoglicemia.

\section{Abstract}

Introduction: Münchhausen syndrome is a subtype of serious and chronic factitious disorder, characterized by the production of pathological lies ("fantastic pseudology") and wandering behavior from doctor to doctor, pretending a physical illness.

Objective: to present the case of a patient with refractory hypoglycemia secondary to the use of exogenous insulin in the context of a Münchhausen syndrome.

Discussion: the patient presented an unusual submission to the hospitalizations and to the diagnostic tests, which confirmed the exogenous administration of insulin to trigger the symptoms. The paranoid features and the mythomania observed are associated with a latent, chronic and delirious state, typical of Münchhausen syndrome.

Conclusion: Münchhausen syndrome is a psychiatric condition that is difficult to diagnose and that constitutes an important challenge for the clinician; it is little recognized, affects the doctorpatient relationship and presents a high cost for the health system due to the use of unnecessary diagnostic and therapeutic procedures. (Acta Med Colomb 2019; 44: 43-46).

Keywords: Münchhausen syndrome, factitious disorder, hypoglycemia.
Dra. Soraya Aparicio-Turbay: Psiquiatra. Departamento de Salud Mental, Fundación Santa Fe de Bogotá. Docente Universidad de Los Andes; Dr. Camilo Alvarado-Castro: Médico Hospitalario. Departamento de Medicina Interna, Fundación Santa Fe de Bogotá; Dr. Efraín Noguera-Alfonso: Psiquiatra. Departamento de Salud Mental, Fundación Santa Fe de Bogotá Docente Universidad de Los Andes. Bogotá D.C. (Colombia).

Correspondencia: Dr. Camilo Alvarado Castro. E-mail: camilo.alvarado5@gmail.com Recibido: 8/XI/2017 Aceptado: 17/I/2019

\section{Introducción}

El 13 de diciembre de 2005 se estrena "Deception", el noveno episodio de la segunda temporada de la serie norteamericana "Doctor House". Relata la historia de Anica, una paciente hospitalizada por crisis convulsiva y hematomas abdominales. En otra ciudad había sido intervenida el año anterior por un supuesto tumor de hipófisis responsable de una Enfermedad de Cushing. Los estudios imagenológicos y de laboratorio no arrojaron resultados, al punto que le sería realizada una nueva intervención transesfenoidal y estudios invasivos de la vena renal. Sin embargo tras realizar una pesquisa exhaustiva de la historia clínica, se desenmascara la naturaleza autoinflingida de los síntomas y lesiones; y se logra determinar un fondo psicodinámico en el caso. El diagnóstico: síndrome de Münchhausen, un subtipo de trastorno facticio grave y crónico, descrito por Asher en 1951 (1), que se caracteriza por la producción de mentiras patológicas ("pseudología fantástica") y una conducta 
errante, de médico en médico, fingiendo una enfermedad física. Es una entidad difícil de diagnosticar por el engaño y la manipulación que la caracteriza (2).

No es un verdadero trastorno somatoforme pero sus características son parecidas a las de los trastornos psicopatológicos de una enfermedad orgánica. Su nombre hace referencia a un personaje histórico: Karl Fiedrich Hieronymus, Barón de Münchhausen (1720-1797) (3), un militar alemán de la caballería del ejército ruso que combatió contra los turcos en 1716 y que se hizo famoso por sus historias de aventuras de caza y guerra, fantásticas e irreales, a lo largo de Europa; Asher lo definía como un caballero que "vagaba de ciudad en ciudad embelleciendo dramáticamente los acontecimientos de su vida". En 1785, Rudolf Erich Raspe recopila y ordena estas historias en la obra literaria "las Aventuras del Barón de Münchhausen".

Los pacientes con síndrome de Münchhausen plantean desafíos para el sistema de salud debido a que buscan obtener el papel de enfermos, que se traduce en la solicitud de múltiples procedimientos y exámenes complementarios innecesarios. Las estimaciones de la incidencia del síndrome van desde 0.6-1.3\% de los ingresos hospitalarios (4), siendo más frecuente en hombres de edad media (a diferencia de los trastornos facticios en general, que son más frecuentes en mujeres).

Son múltiples las publicaciones relacionadas con los distintos patrones de conducta, que han sido incluidas bajo el concepto "espectro Münchhausen" (5) como lo son: infecciones autoprovocadas (abscesos por inyección de materia fecal u otras sustancias), fiebre de origen indeterminado, retraso en la curación de heridas por autolesión, hipoglicemias por administración exógena de medicamentos, anemia, hemoptisis masivas, hemorragias por sobre-anticoagulación, crisis y pseudocrisis convulsivas, aparición de lesiones cutáneas, incluso consumo masivo de metilxantinas (para provocar arritmias cardiacas) y yoduros (para provocar tormentas tiroideas).

Generalmente tienen un nivel intelectual alto y un conocimiento minucioso sobre las prácticas médicas, llegando a manipular de tal modo, que consiguen hospitalizaciones e intervenciones mayores que pueden ser riesgosas, como las reportadas por Gaudino y colaboradores en 2013 (6), donde dos pacientes fueron intervenidos en varias ocasiones con esternotomía mediana en búsqueda de segmentos aórticos comprometidos por disección. Se ha propuesto una explicación a través de teorías psicodinámicas que postulan una reactivación de conflictos inconscientes marcada por resentimiento contra médicos y hospitales, donde la apariencia de desamparo del enfermo, busca venganza mediante el engaño y la hostilidad. Dicho resentimiento aflora una vez se descubre el engaño o se sospecha sobre la veracidad de los síntomas, incluso puede tornarse en una conducta agresiva y violenta por parte del paciente. Otros autores afirman que el entorno hospitalario brinda un sustituto de la figura parental ambivalente, protectora, controladora y agresiva (7).

\section{Presentación del caso}

Paciente de 26 años de edad, sexo masculino, estudiante universitario, de origen y procedencia Bogotá, que ingresó al servicio de urgencias por cuadro clínico de 24 horas de inicio consistente en somnolencia y traumas contusos secundarios a aparente caso de robo y secuestro. Refirió que fue abordado por individuos que se movilizaban en una camioneta y fue obligado a subir en ella, le fue suministrada una sustancia (que obligaron a ingerir) y que le hizo "perder el conocimiento" razón por la cual no recuerda otros hechos relacionados. Fue recogido por una ambulancia y traído a la institución, donde se registró glucometría al ingreso de $38 \mathrm{mg} / \mathrm{dL}$, por lo cual se inició reposición con dextrosa, y se alcanzó glicemia de $160 \mathrm{mg} / \mathrm{dL}$.

Como antecedentes personales solo refirió migraña clásica, negó consumo de bebidas alcohólicas, tabaco o alucinógenos. Al examen físico de urgencias no se evidenciaron alteraciones diferentes a los síntomas neuroglucopénicos (somnolencia) y autonómicos (sudoración). Se solicitaron exámenes complementarios (hemograma, hepatograma, gasometría arterial, pruebas de función renal, electrolitos séricos), toxicológicos (niveles de etanol y metanol, benzodiacepinas, escopolamina, fenotiazina) e imagenológicos (tomografía de cráneo). En la hora posterior al ingreso del paciente, nuevamente cursa con cuadro de somnolencia y se solicitó glucometría donde se observó resultado de 20 $\mathrm{mg} / \mathrm{dL}$ que requirió nuevamente reposición con dextrosa. Los exámenes complementarios y toxicológicos iniciales fueron normales.

Durante su estancia en el servicio de urgencias se observaron glucometrías menores a $40 \mathrm{mg} / \mathrm{dL}$, razón por la cual se sospechó hiperinsulinismo endógeno como causa de hipoglicemia refractaria. Los estudios toxicológicos para benzodiacepinas, atropínicos y alcohol etílico y metílico fueron negativos. Finalmente se decidió interconsulta al servicio de endocrinología. Al interrogatorio con los familiares se documentó que dos meses antes había consultado en otra institución por cuadro similar secundario a robo y que presentó hipoglicemia refractaria, sin embargo no fue estudiado debido a que solicitó egreso voluntario.

El servicio de endocrinología decidió hospitalización y solicita estudio de insulinoma donde se observaron niveles de insulina de $21.45 \mathrm{uU} / \mathrm{mL}$ y péptido $\mathrm{C}$ menor de 0.1 $(\mathrm{nmol} / \mathrm{L})$. Se solicitaron niveles de sulfonilureas y betahidroxibutirato y nuevamente se corroboraron niveles de insulina y péptido $\mathrm{C}$. Al día siguiente al ingreso a hospitalización se presentó nuevo episodio de hipoglicemia de $46 \mathrm{mg} / \mathrm{dL}$ con síntomas neuroglucopénicos que requirió reposición con dextrosa. La tomografía axial computarizada de abdomen no reveló lesiones focales solidas, quísticas ni hipervasculares a nivel de páncreas. Ante sospecha de hiperinsulinismo de origen exógeno se decidió interconsulta al servicio de psiquiatría por sospecha de trastorno facticio debido a que el insulinoma no se relaciona con cifras elevadas de insulina sérica y el péptido $\mathrm{C}$, el cual debería estar elevado en caso 
de secreción endógena debido a la respuesta fisiológica supresora del péptido $\mathrm{C}$ en presencia de hipoglicemia.

A la entrevista psiquiátrica se intentaron abordar las circunstancias del evento donde añadió que se encontraba realizando trámites en su universidad y que una vez sale a la calle fue abordado por individuos (no recordó cuántos), quienes lo metieron al baúl del vehículo (no recordó marca ni color), posteriormente fue obligado a salir a un lugar desconocido ("espesa vegetación") donde fue golpeado y obligado a "ingerir 15-20 pastillas azules o moradas, bajo amenaza de ser herido con arma de fuego".

Refirió que posteriormente se despertó en la ambulancia sin saber distinguir si se encontraba soñando o era realidad. Relató en la entrevista que se encontraba en estudios médicos por posible "intoxicación por metales pesados" y "enfermedades del colágeno". Una vez se intentó abordar el tema de la posible administración exógena de insulina (confrontación) el paciente se torna hostil, irritable y evasivo razón por la cual se suspendió la entrevista.

Al examen mental directo se describieron ideas de referencia relacionadas con el hecho delictivo, con rasgos paranoides, sin actividad alucinatoria y afecto plano. Ante trastorno de la personalidad de rasgos paranoides, posiblemente relacionado con la construcción de identidad falsa y tendencia al engaño, se decide inicio de manejo con ansiolíticos a dosis bajas (clonazepam) y psicoterapia. Al tercer día de hospitalización en horas de la madrugada se documentó glucometría de $40 \mathrm{mg} / \mathrm{dL}$ con síntomas neuroglucopénicos que requirió reposición con dextrosa. Ante esta nueva hipoglicemia, el servicio de endocrinología sugiere la realización de ecoendoscopia y test de ayuno, en éste último no se observaron síntomas neuroglucopénicos ni autonómicos.

Nuevamente se intenta entrevista psiquiátrica para realizar confrontación; sin embargo, el paciente se torna hostil, no colabora y refiere "no me gusta que se metan en mi vida". En el día cuatro de hospitalización los familiares entregan un papel al psiquiatra donde escribió "abrir solo en caso de algo grave" y en el sugería que donen su cuerpo en caso de muerte. Al intentar abordarlo sobre el significado de este escrito se observa una actitud distante, fría, impermeable y responde "es que de las experiencias con mi enfermedad se aprende". Sin embargo, niega pensamientos de muerte, niega ideación o planificación de actos suicidas.

Se documentó la posibilidad de construcción de personalidad irreal con rasgos mitómanos, continuándose el manejo ansiolítico y la compañía permanente ante posibilidad de autoagresión. En el día cinco de hospitalización se comprobaron niveles de insulina sérica superiores a $13.3 \mathrm{uU} /$ $\mathrm{mL}$ en las muestras sanguíneas tomadas durante las crisis hipoglicémicas. Los estudios de control permitieron detectar péptido C de $1.68 \mathrm{nmol} / \mathrm{Lt}$ que no concordó con los niveles de insulina superiores a $200 \mathrm{uU} / \mathrm{mL}$, lo que indicó administración exógena de insulina. Dicho día se documentó tendencia evasiva a la entrevista, refirió que no necesitaba apoyo psicoterapéutico, lo que permitió determinar introspección nula. Ante cuadro clínico de características delirantes mitómanas, de evolución larvada relacionadas con necesidad de adquirir el papel de enfermo, que incluso se acompañó de conductas riesgosas para su integridad, se diagnostica trastorno facticio de subtipo síndrome de Münchhausen.

Se decidió remisión a clínica psiquiátrica para manejo intramural, sin embargo el paciente y los familiares se niegan a su traslado y deciden solicitar egreso hospitalario, a pesar de las recomendaciones del equipo multidisciplinario y los riesgos relacionados con el trastorno.

\section{Discusión}

Los criterios DSM-V para trastorno facticio incluyen: fingimiento o producción intencionada de signos o síntomas físicos y psicológicos, el sujeto busca asumir el papel de enfermo y se observa ausencia de incentivos externos para explicar el comportamiento (7). Sus categorías incluyen trastornos facticios con predominio de signos y síntomas psicológicos, trastornos facticios con predominio de signos y síntomas físicos (como el síndrome de Münchhausen) y un trastorno mixto definido por síntomas y signos psicológicos y físicos.

Las características principales del síndrome de Münchausen son: presencia de enfermedad recurrente, fingida o simulada, con tendencia a la peregrinación (viajeros y errantes) y pseudología fantástica, una forma de mentira patológica caracterizada por historias exageradas e irreales, con estilo histriónico (6). Los rasgos de personalidad observados en el paciente, permitieron documentar un relato dramático y exagerado, que se acompañó de vaguedad y circunstancialidad una vez se intenta confrontar en forma detallada los hechos.

La pseudología fantástica, descrita en el síndrome de Münchhausen, se observó en el paciente a tal punto que incluyó la construcción de un hecho irreal basado en el secuestro, donde él mismo se consideraba la víctima. El paciente presentó una sumisión inusual a las hospitalizaciones y a las pruebas diagnósticas, las cuales confirmaron la administración exógena de insulina para desencadenar los síntomas. Los rasgos paranoides y la mitomanía observada se asocian a un estado larvado, crónico y delirante. A diferencia de la simulación no se observó intencionalidad de ganancia externa, económica o de eludir responsabilidades laborales o judiciales (8). Stephen y colaboradores (9) reportaron un caso similar, en un paciente de 25 años, con cuadro clínico de hipoglicemia refractaria y midriasis derecha, donde se pudo determinar administración exógena de insulina y pilocarpina oftálmica.

A menudo se describe que los afectados por este trastorno facticio, una vez llegan a ser confrontados o se descubre el engaño del suceso, tienden a demostrar hostilidad y agresividad. Su conducta puede llegar incluso a hacerles incurrir en falsificación de historias clínicas y uso de diferentes identidades para evitar ser descubiertos. En una revisión sistemática de Kenedi y colaboradores (10), en 2011 se 
propone una serie de baterías diagnósticas que incluyen exámenes de laboratorio, imágenes, procedimientos y estudios anatomopatológicos para desenmascarar sistemáticamente los trastornos facticios.

El diagnóstico diferencial se debe realizar con otros trastornos. El trastorno de somatización presenta múltiples quejas físicas que dan lugar a búsqueda del tratamiento o disminución de la función, sin embargo tienen menor tendencia a la mentira y no causan su enfermedad intencionalmente. Los simuladores tienen como objetivo la ganancia secundaria, una recompensa tangible más allá de una ganancia primaria (beneficio emocional de considerarse paciente). No se someten a procedimientos invasivos y dolorosos.

Otro aspecto importante, es que a diferencia de los simuladores y de los trastornos somatomorfos, los pacientes con síndrome de Münchhausen tienen poca o ninguna capacidad de controlar su comportamiento autodestructivo. Iarsen describe las posibles implicaciones médico-legales en que pueden incurrir estos pacientes debido a sus actuaciones y la responsabilidad del equipo de salud y de la familia.

Las características delirantes larvadas hacen difícil el manejo del síndrome de Münchhausen y ensombrecen el pronóstico.

\section{Conclusiones}

El síndrome de Münchhausen es una condición psiquiátrica difícil de diagnosticar y que constituye un importante reto para el clínico, es poco reconocido, afecta la relación médico-paciente y presenta un alto costo para el sistema de salud debido al uso de innecesaria de baterías diagnósticas y procedimientos terapéuticos.
Se debe sospechar en pacientes que ocultan información de la historia clínica, que insisten en atención constante por parte del personal asistencial para aliviar su sufrimiento y presentan una conducta demandante por medicamentos, procedimientos diagnósticos y pruebas de laboratorio.

En la actualidad, el fácil acceso a la información a través de múltiples medios de comunicación, puede aumentar la versatilidad de las presentaciones clínicas del síndrome. El papel del psiquiatra es ayudar al equipo de tratamiento primario, proporcionar un seguimiento que evite procedimientos innecesarios, autolesiones y reacciones hostiles del paciente hacia el personal asistencial.

\section{Referencias}

1. Asher R. Munchausen's syndrome. Lancet 1951;1:339-341.

2. Gheis S, Mayer R. Münchhausen syndrome. Clin Ter 2000; 151(5):351-5.

3. Olry R. Baron Münchhausen and the syndrome which bears his name: history of andearing personage and of a strange mental disorder. Vesalius 2002; 8(1):53-7.

4. Fliege H, Grimm A, Eckhardt-Henn A, Gieler U, Martin K, Klapp BF. Frequency of ICD-10 factitious disorder: survey of senior hospital consultants and physicians in private practice. Psychosomatics 2007;48(1):60-64

5. Huffman JC, Stern TA. The diagnosis and treatment of munchausen's syndrome. Gen Hosp Psychiatry 2003;25:358-63.

6. Gaudino M, Nasso G, Romano V, Pargiola C. Acute aortic pathology, Munchausen síndrome and confirmation bias. Journal of emergency medicine 2013; 45 (6): 183-186.

7. Jones R. Trastornos Facticios. En: Kaplan H,Sadock B. Tratado de Psiquiatría. $10^{\mathrm{a}}$ ed. Buenos Aires: Inter-Médica; 2009, pp. 1197-1205

8. Turner J and Reid S. Munchausen Syndrome. Lancet. 2002. 359:346-49.

9. Stephen W, Richards J. Munchausen Syndrome presenting acutely in the emergency department. Journal of emergency medicine 2000; 18 (4): 417-420

10. Kenedi C, Shirey K, Hoffa M, Zanga J, Lee J, Harrison J. Laboratory diagnosis of factitious disorder: a systematic review of tools useful in the diagnosis of Munchausen's syndrome. Journal of the New Zealand Medical Association 2011; 124: 66-81 